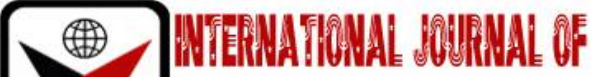

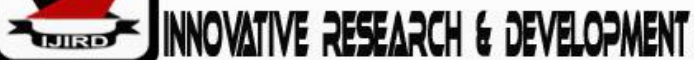

ISSN 2278-0211 (Online)

\section{Creative Designing of Fabric Patterns by Tie-Dyeing with Synthetic Dyes}

\author{
Nelima Barasa \\ Lecturer, Department of Physical sciences, Rongo University, Kenya \\ Francis Ongachi Olal \\ Senior Lecturer, Department of Physical sciences, Rongo University, Kenya
}

\begin{abstract}
:
Tie and dye are a resist method of fabric decoration where patterns are skillfully introduced on plain fabrics through tying and dyeing with synthetic dyes. Hot synthetic dyes were used in this study due to their advantage in terms of color variety, brightness and fastness. This study produced fabric pattern designs by tie and dyeing with hot water dyes which are good in terms of color fastness. Different types, forms, techniques, and processes of creating unique patterns by tie and dyeing was done. Basic tie-dyeing methods twisting, folding, pleating, wrapping, crumbling and sewing were then used to create new patterns when dyeing each color. Though tie and dye as a technique is familiar to many people, exploration in terms of the unique fabric patterns design has not been done especially in Kenya thus making us to get the tie-dyed fabrics from the international market. This paper therefore creatively introduced unique patterns on fabrics through folding, twisting, crumpling and tie and dye. The fabric patterns formed are very unique compared to what is available in the Kenyan market. The tie-dyed fabrics can be used in designing and developing fashion items like men's shirts, ladies wear, ladies hand bags and scuffs. The methodology involved aims at create unique fabric patterns which was done through folding, twisting, crumpling and tying, followed by preparation of the dye bath and then dyeing the prepared fabrics.
\end{abstract}

Keywords: Synthetic dye, Tie and Dye, Fabric patterns, Creative Designing, Hot water dyes

\section{Introduction}

Tie and dye is a resist method of fabric decoration where patterns are skillfully introduced on plain fabrics through tying and dyeing with synthetic dyes. Hot synthetic dyes are used due to their advantage in terms of color variety, brightness and fastness (Behera \& Khandual, 2017). Tie-dye describes a pattern of color made by preventing the dye from reaching some areas of the fabric while dyeing others (Saowanit, 2000). Folding, tying, swirling and crumpling inhibit the flow of the dye to some areas of the fabric (Goetz et al., 2008).

Vat dyes, including indigo, are a third class of dyes that are effective on cellulosic fibers and silk. Vat dyes are a class of dyes that are classified as such because of the method by which they are applied. Vat dyeing is a process that refers to dyeing that takes place in a bucket or vat.

Vat dyes have excellent fastness in all areas as well, especially to chlorine and bleach. However, if not properly applied, they may crock (Goertz, 2008). Vat dyes are insoluble in water in their unreduced form, and the vat dye is chemically reduced before they can be used to color fabric. This is accomplished by heating the dye in a strongly basic solution of sodium hydroxide (lye) or sodium carbonate (caustic potash) containing reducing agent such as sodium hydrosulfite or thionite dioxide. The fabric is immersed in the dye bath, and after removal the vat dye oxidizes to its insoluble form, binding with high wash-fastness to the fiber. However, vat dyes, and especially indigo, must be treated after dyeing by 'soaping' to prevent the dye from rubbing (crocking) off (Wisanu, 2010). Vat dyes can be used to simultaneously dye the fabric and to remove underlying fiber-reactive dye (i.e., can dye a black cotton fabric yellow) because of the bleaching action of the reducing bath. Strong bases like lye should be avoided when tie dying for safety purposes (Mayusoh, 2015)

\subsection{Creative Design Patterns}

Creative designing of fabric patterns through tie and dye, is a skillful and artistic processes that produces unique designs in terms of color and design. Currently, the Kenyan market is limited in terms of the type of tie-dyed fabric. The available tie-dyed fabrics are more feminine in terms of color and design. To achieve unique but creative designs, lots of practice and creativity is required. The pattern of the folds, swirling and twisting etc. and where the colors are squirted dictates the resulting design. With experience, the end result can be predicted and controlled to some extent, but surprise is part of what makes tie-dye an exciting and interesting art form and there will be plenty of surprises. 
The tie-dye technique involves various methods of folding fabrics, tying and binding them, then dyeing the fabric in a dye bath. Parts of the fabric do not receive the dye because of their bindings and in this way, designs are created. Tiedye can be used to create a wide variety of designs on fabric, from standard patterns such as the spiral, peace sign, diamond, and the marble effect to beautiful works of art (Saowanit, 2000). Using techniques such as stencils (a la screenprinting using dyes or discharge pastes), clamped-on shaped blocks, and tritik (stitching and gathering), tie-dye can produce almost any design desired.

\section{Material and Method}

The study finds technical and artistic knowledge about designing fabric patterns by tie and dye using vat dyes. This is a study of creativity in terms of design, techniques and the processes of dyeing.

\subsection{Material Required for Tie Dying}

- Fabric the best is $100 \%$ white cotton, silk, unbleached muslin (calico), rayon, nylon, velvet, organdie

- Hand gloves

- Nose protectors

- Vat dyes

- Heat source

- Container for dye solution, e.g. can, basket

- Rubber or plastic gloves $\backslash$

- Rubber bands

- String/cord

- Stick to stir the dye bath

- Caustic soda and sodium hydro sulphite

- Spoons for measuring

- Water

- Working surface

- Paper to cover working area old newspaper or polythene

\subsection{Method}

\subsubsection{Chevron}

The fabric was laid flat on a table.

The fabric was folded into two halves as shown

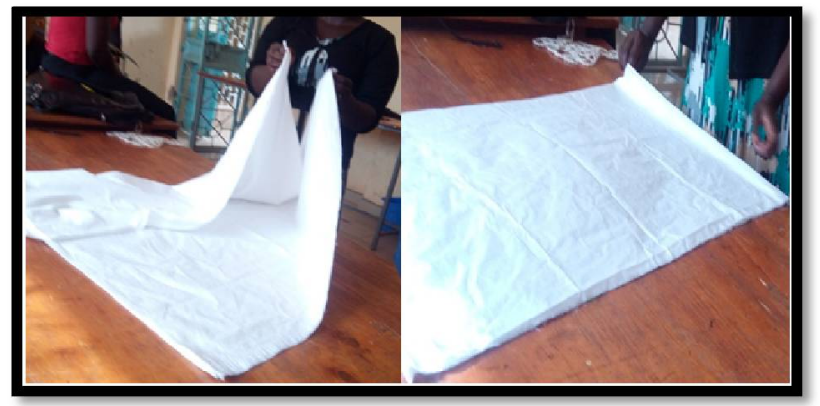

Figure 1: Laying of Faric and Folding In Half

From one corner it is accordion pleated diagonally through to the end as shown below

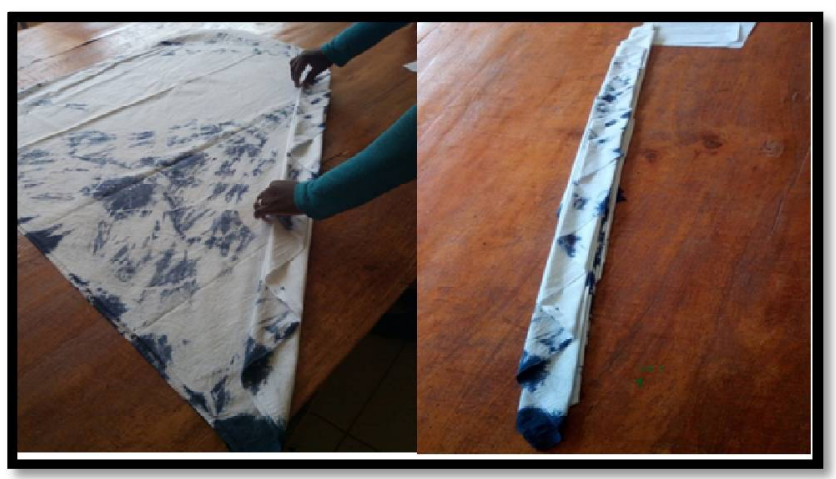

Figure 2: Accordion Pleating of the Fabric

The pleated piece was bound straight across. 


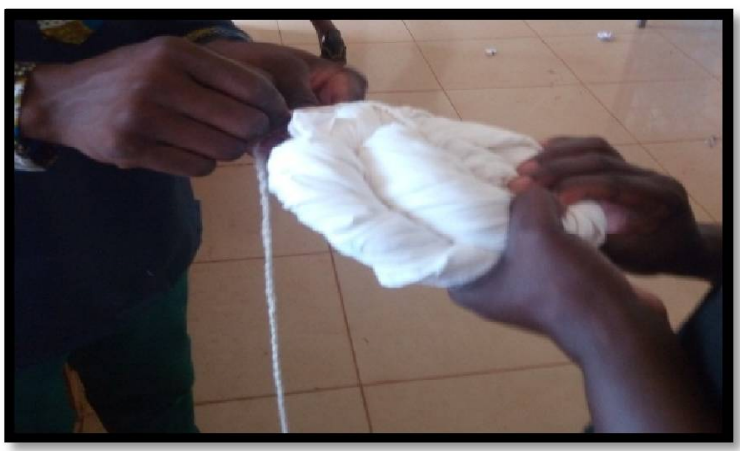

Figure 3: Binding the Accordion Pleated Piece

The accordion pleated piece was immersed in clean cold water for 5 minutes, removed and all water squeezed out. It was dyed in a prepared dye bath for 5-10 minutes, removed and rinsed in clean water and the cords untied.

\subsubsection{Samosa Folding}

The fabric was laid flat on a table.

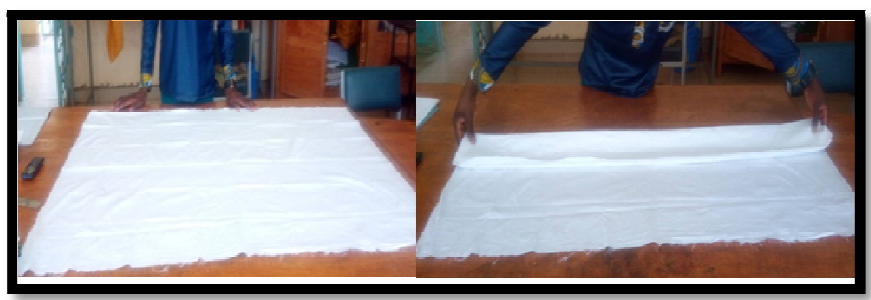

Figure 4: Laid Fabric and Folding

Start folding samosa shape folds from one end heading to the other end.

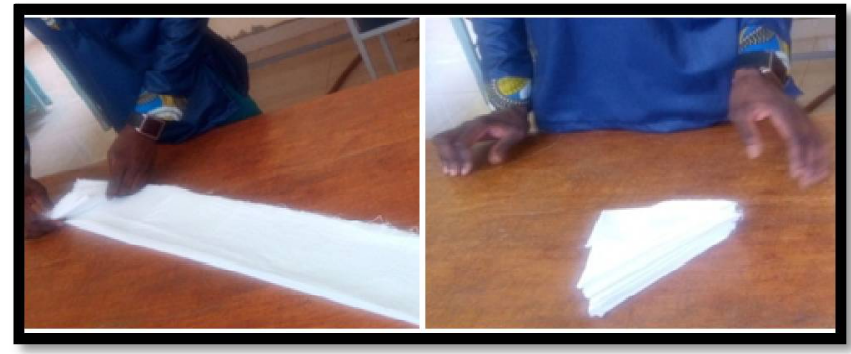

Figure 5: Samosa Shape Pleating

The samosa shaped piece was bound at corners with the cord to prevent the dye from penetrating.

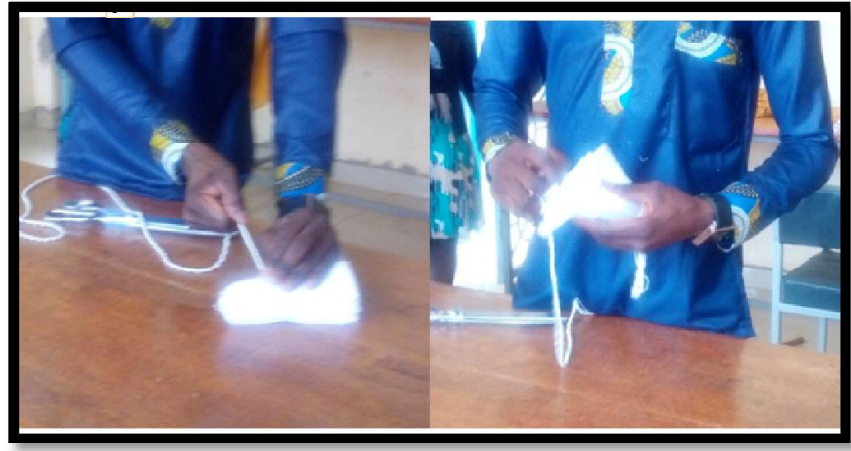

Figure 6: Binding of the Samosa Pleated Piece

The piece was immersed in clean cold water to increase the dye affinity and then all water squeezed out after 5 minutes. The contents were mixed in a basin ready for dying. 


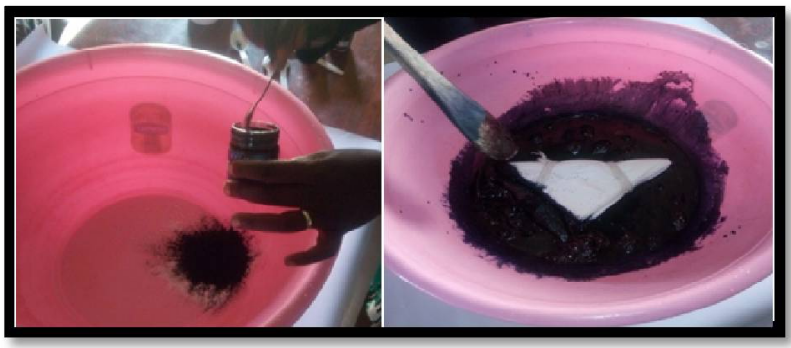

Figure 7: Dye Preparation and Dying

It was allowed to stay in the dye for 5-10 minute, removed and rinsed in clean water to remove excess dye and the dyed piece untied.

\subsubsection{Swirl}

Fabric was pinched at the spiral point and twisted in a circle to form a flat spiral.

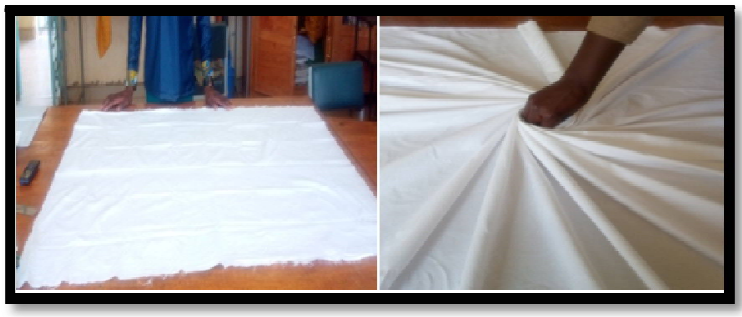

Figure 8: Laying the Fabric Flat on the Table and Swirling

The spiral was bound with three rubber bands that crossed each other and form six equal pie pieces.

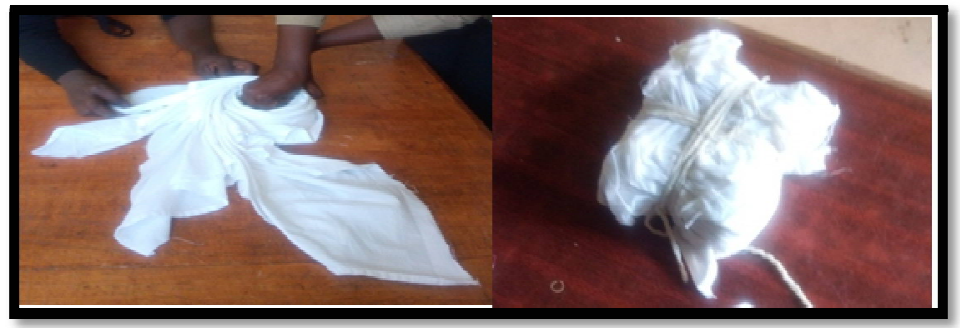

Figure 9: Bound Swirled Piece Ready for Dying

Using a spoon, the dye was applied to each wedge of the fabric making sure each side was dyed.

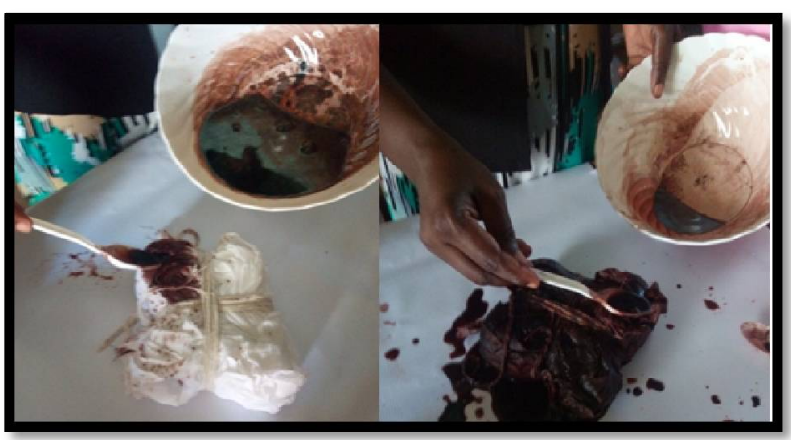

Figure 10: Dying the Bound Piece

It was rinsed in cool water until the water ran clear; washed and dried separately using hot water and a little laundry soap.

\subsubsection{Box Folding}

Fabric was ironed to remove creases.

Fabric was folded in half and laid flat on the table. 


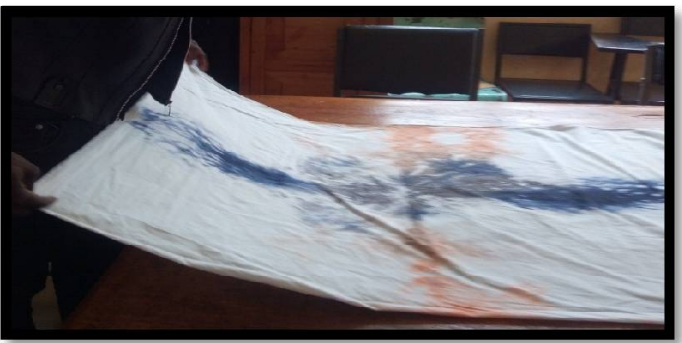

Figure 11: Folding Fabric on a Flat Surface

Layers were folded along the fabric to the end according to the size of the boxes to be formed.

Sizable squares were folded alternating on the sides to the end and bound in four sections ready for dyeing.

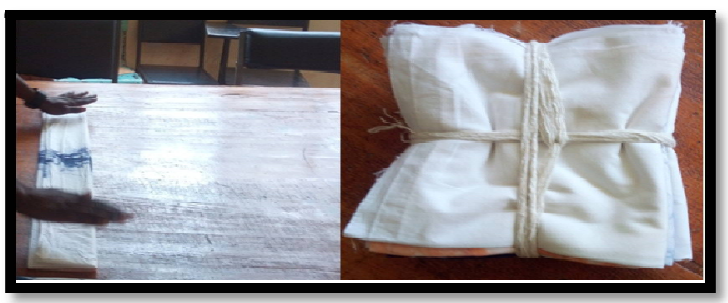

Figure 12: Folding the Piece and Binding

The tied piece was immersed in clean water for 5 minutes, removed and all the water and dye squeezed out.

\section{Results and Discussion}

In this study creativity in terms of technique and use of colors of tie and dye was applied. This led to four different patterns that were achieved through folding and swirling. Vat dyes were used because of their color fastness and variety of colors. The tie-dyed fabrics can be used for making clothing, fashion accessories and soft furnishings.

The study finds technical and artistic knowledge about designing fabric patterns by tie and dye using vat dyes. This is a study of creativity in terms of design, techniques and the processes of dyeing.

\subsection{Chevron}

In chevron technique the result is shown in Figure 13

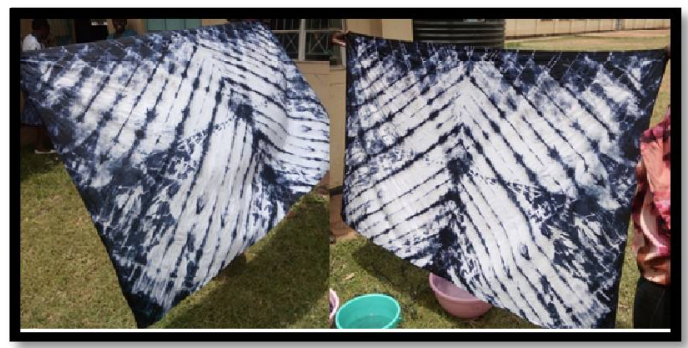

Figure 13: Tie Dyed Chevron Technique Fabric

\subsection{Samosa Folding}

From Dyed samosa design technique, the result is shown in Figure 14

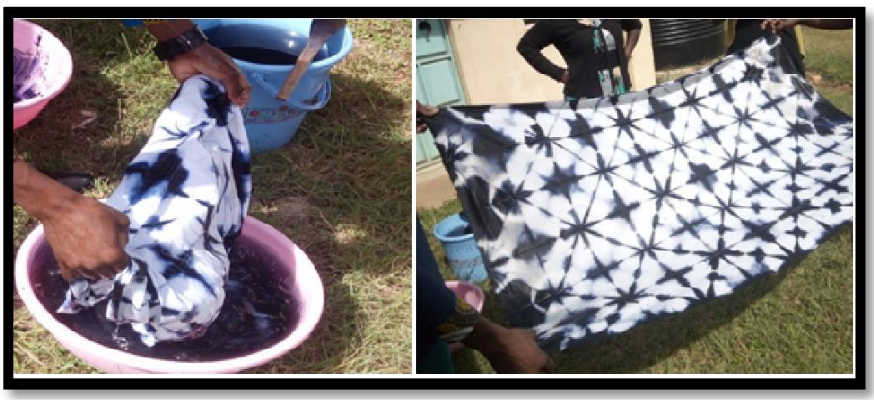

Figure 14: Dyed Samosa Design 


\subsection{Swirl}

In swirl technique the result is shown in Figure 15

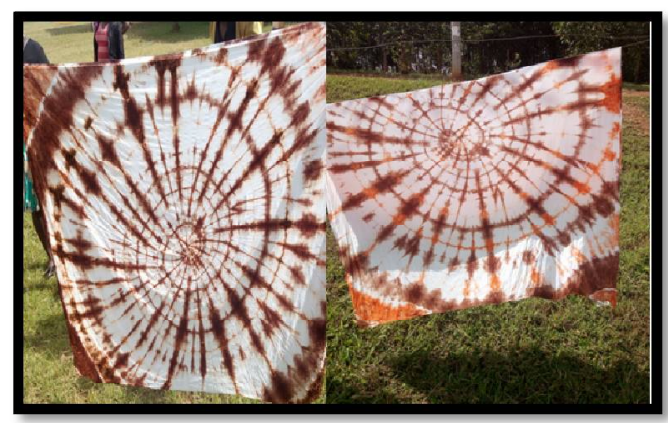

Figure 15: Dyed Swirl Design Fabric

\subsection{Box Folding}

In box folding technique the result is shown in Figure 16

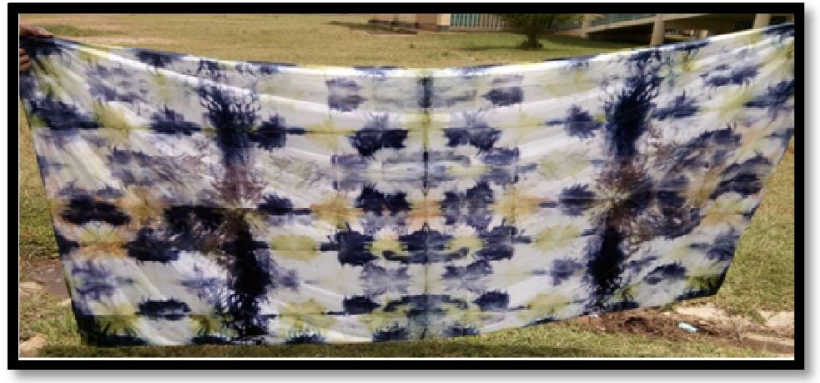

Figure 16: Dyed Box Folded Design Fabric

\section{References}

i. $\quad$ Behera, S., \& Khandual, A. (2017). Tie Dye Textiles in Fashion Domain. International Journal of Engineering and Technical Research , 28-51.

ii. Goetz, C. (2008). Textile Dyes: Techniques and their effects on the enviroment with a textile Dye: Techniques and their effects on the Enviroment with a recommendation for Dyers concerning the Green Effect. Liberty University

iii. Mayusoh, C. (2015). The art of designing, fabric pattern by tie-dyeing with Natural dyes. Social and Behavioural Sciences (pp. 1472-1480). Greece: Elsevier.

iv. Nwadiokwu, E.S., Ezeanyanaso, C.S., Akinboade, D.A., Akubueze, E.U., Muniru, O. S. and Igwe, C.C. Improvement of Dying Process Parameters in the Production of Tie and Dye Cotton Fabric.

v. Saowanit Karnchanarat. (2000). Designing tie-dye patterns with chemical and natural dyes (pp.23). Nakhon Sri Thammarat: Nakhon SriThammarat Rajabhat University

vi. Wisanu Dathong. (2010) http://www.nstda.or.th online 